\title{
Actividad antimicrobiana de cuatro variedades de plantas frente a patógenos de importancia clínica en Colombia
}

\author{
Antimicrobial activity of four varieties of plants against pathogens clinical \\ significance in Colombia
}

Título abreviado: Plantas y actividad antimicrobiana en patógenos humanos

Cristian Nicolás Rodríguez Pava ${ }^{1}$, Andrés Gabriel Zarate Sanabria ${ }^{1}$, Ligia Consuelo Sánchez Leal ${ }^{2}$

\section{Resumen}

Objetivo. Evaluar la actividad antimicrobiana de los extractos de las plantas Bauhinia sp., Sambucus nigra, Eichhornia crassipes y Taraxacum officinale frente a patógenos de importancia clínica. Método. La metodología incluyó la adquisición, secado, maceración, molienda, preparación de los extractos crudos etanólicos y concentración por rotaevaporación, análisis fitoquimico y se separaron las fracciones por cromatografía en capa fina. Las pruebas antimicrobianas se realizaron con diferentes concentraciones de los extractos según las indicaciones de Clinical and Laboratory Standars Institute. Los microorganismos utilizados fueron Enterococcus faecium resistente a vancomicina, Streptococcus pneumoniae, Klebsiella pneumoniae con presencia de KPC, Providencia rettgeri con presencia de ESBLs, Pseudomonas aeruginosa, Enterobacter cloacae, Escherichia coli, Staphylococcus aureus $\beta$-lisina y Candida albicans. Resultados. Las cromatografías permitieron comprobar la presencia de flavonoides, terpenos, saponinas, fenoles, quinonas y alcaloides que han sido reportados con actividad antimicrobiana. En los ensayos de susceptibilidad antimicrobiana se encontró que los extractos presentaban diversos grados de inhibición frente a los microrganismos de estudio, siendo el más eficaz los tallos de T. officinale. Conclusión. Se puede concluir que los extractos vegetales podrían ser una alternativa de tratamiento para infecciones nosocomiales.

Palabras claves: Resistencia bacteriana, plantas medicinales, extractos orgánicos, actividad antimicrobiana, ensayos de susceptibilidad, análisis fitoquímicos.

\section{Abstract}

Objective. To evaluate the antimicrobial activity of extracts of plants Bauhinia sp., Sambucus nigra, Taraxacum officinale and Eichhornia crassipes against clinically important pathogens. Method. The methodology included the acquisition, drying, soaking, grinding, preparing ethanolic crude extracts and concentration by rotary evaporation; phytochemical analysis, visualization by thin layer chromatography. Antimicrobial tests were performed with different concentrations of extracts as indicated by the Clinical and Laboratory Standards Institute. The microorganisms used

1. Bacteriólogo y Laboratorista Clínico. Investigador auxiliar grupo CEPARIUM. Universidad Colegio Mayor de Cundinamarca.

2. Docente de tiempo completo, Facultad Ciencias de la salud, Universidad Colegio Mayor de Cundinamarca. Investigadora principal Grupo CEPARIUM. 
were Enterococcus faecium vancomycin-resistant, Streptococcus pneumoniae, Klebsiella pneumoniae with KPC, Providencia rettgeri presence of ESBLs, Pseudomonas aeruginosa, Enterobacter cloacae, Escherichia coli, Staphylococcus aureus $\beta$-lysine and Candida albicans. Results. Chromatographies allowed checking the presence of flavonoids, terpenes, saponins, phenols, quinones and alkaloids have been reported having antimicrobial activity. In antimicrobial susceptibility tests it found that extracts showed varying degrees of inhibition against microorganisms study, the most effective stems T. officinale. Conclusion. It can be concluded that plant extracts may be alternative in the treatment of nosocomial infections.

Keywords: Bacterial resistance, medicinal plants, organic extracts, antimicrobial activity, susceptibility testing, phytochemical analysis.

\section{Introducción}

En los últimos años se han realizado investigaciones dirigidas a buscar nuevas terapias antimicrobianas como opciones alternas a los tratamientos con antibióticos conocidos $(1,2)$, debido a la alta tasa de resistencia que presentan los patógenos microbianos en todo el mundo. Este importante problema de salud pública involucra a todos los países y estamentos mundiales debido a su impacto en la salud, como en el costo-beneficio que implica tratar estas patologías (1).

Estudios realizados por la Organización Mundial de la Salud (OMS), Comunidad Andina de Naciones (CAN) y la Universidad Nacional de Colombia (3), entre otros estamentos nacionales e internacionales (4), establecen que mediante la bioprospección y aprovechando los recursos naturales, se podrían diseñar alternativas de tratamiento (5-6). Uno de los organismos con los cuales se está trabajando para obtener nuevos compuestos antimicrobianos son las plantas.

El género Bauhinia está ampliamente distribuido (familia: Caesalpiniaceae) caracterizada por ser árboles, arbustos trepadores y de fácil adaptación. Ciertas especies de Baubinia tienen una larga historia de usos medicinales tradicionales (7). La planta Bauhinia sp., conocida comúnmente como "pata de vaca” es ampliamente utilizada en la medicina tradicional. Su corteza, raíces, hojas, semillas y flores se usan por sus propiedades medicinales. Se ha uti- lizado en dispepsia, bronquitis, lepra, úlcera; para prevenir la obesidad, como astringente, tónico y antihelmíntico (8).

Otra planta usada por nuestros ancestros es Sambucus nigra (Familia: Caprifoliacea), conocida como "Sauco", una planta arbustiva de origen europeo. Sus frutos, hojas, corteza y raíces son ampliamente utilizados en la medicina tradicional por sus propiedades terapéuticas identificadas (diuréticas, antipiréticas, antisépticas, cicatrizantes, antiinflamatorias y contra los problemas respiratorios) (9-10).

Eichhornia crassipes, conocida comúnmente como "buchón de agua" o "jacinto de agua" y originaria de Brasil, es una planta acuática perteneciente a la familia Pontideriaceae. El buchón de agua es una planta flotante, que se caracteriza por formar densas poblaciones en el agua y el barro (11), puede tener efectos negativos sobre el medio ambiente, la salud humana y desarrollo económico por la invasión que realiza en los cuerpos de agua (12). Estudios han demostrado la eficacia del extracto crudo de Eichhornia crassipes y varias fracciones frente a bacterias Gram positivas y Gram negativas, encontrándose diversas actividades antibacterianas atribuidas a algunas de las fracciones aisladas (13-14).

Taraxacum officinale es una planta herbácea perenne de la familia Asteraceae, conocida comúnmente como "diente de león". La planta crece en las regiones templadas del mundo. Esta planta es utilizada 
tradicionalmente para la indigestión, retención de líquidos y para hepatitis (14). Taraxacum officinale se ha demostrado que posee compuestos con una amplia variedad de actividad antimicrobiana frente a patógenos tanto Gram positivos como Gran negativos (15).

Esta investigación buscó establecer el efecto y la posible actividad antimicrobiana de los extractos obtenidos a partir de Bauhinia sp., Sambucus nigra, Eichhornia crassipes y Taraxacum officinale con potencial antimicrobiano frente a siete bacterias y una levadura de importancia clínica en Colombia, mediante pruebas de susceptibilidad en placa y perfil fitoquímico.

\section{Materiales y métodos}

\section{Material vegetal}

Sambucus nigra, Taraxacum officinale, Bauhinia sp., se adquirieron por compra en Bogotá D.C., Colombia y Eichhornia crassipes se obtuvo por donación del dueño de la finca "El Jardín" ubicada en la vereda de Chicaque, Municipio de San Antonio del Tequendama, departamento de Cundinamarca, Colombia. La identificación taxonómica de las cuatro plantas se realizó en el Herbario del Instituto de Ciencias Naturales de la Universidad Nacional de Colombia.

La selección del material vegetal se hizo de acuerdo con lo recomendado por Brango y colaboradores (16) y se eliminó el que presentaba manchas, quemaduras, daño de tejido por hongos o bacterias. Las partes utilizadas de cada planta fueron: tallos y hojas para Bauhinia sp., Eichhornia crassipes y Taraxacum officinale y para Sambucus nigra hojas, tallos, flores y bayas. Las partes de las plantas fueron tomadas según disponibilidad al momento de su compra y recolección respectivamente.

\section{Secado y molienda}

Las hojas, tallos y flores, según correspondió de cada planta, se secaron, separando cada parte, a una temperatura promedio de $42^{\circ} \mathrm{C}$ por un período de 4 días. Una vez secas las partes de las plantas, se pro- cedió al triturado (16). Para las bayas de $S$. nigra se procedió a extraer el zumo y realizar su respectiva extracción (16).

\section{Extracción con solventes orgánicos}

Para realizar la extracción etanólica de los tallos, hojas y flores se siguió el protocolo de Tello y colaboradores (17), las diferentes partes de las plantas trituradas (250g por cada parte de las plantas) se depositaron en recipientes de vidrio y se procedió a agregar $500 \mathrm{ml}$ de etanol en cada recipiente y se dejó en obscuridad y sellado por ocho días. Posterior a este periodo se procedió a filtrar todo el material mediante un sistema al vacío mediante embudo de Büchner, luego se concentró en el rotaevaporador (Eyela ${ }^{\oplus}$ ) a $40^{\circ} \mathrm{C}$ y $175 \mathrm{mbar}(17)$.

La extracción del zumo de las bayas de Sambucus nigra se realizó con butanol debido a que los compuestos que se hallaban en esta parte de planta son en su mayoría polares y este solvente permite su mayor recuperación en sustancias acuosas (16).

\section{Análisis fitoquímico}

Para realizar el análisis fitoquímico se partió del extracto etanólico concetrado y se evaluaron los siguientes componentes según el protocolo de Harborne y colaboradores (18).

1. Quinonas. $1 \mathrm{mg}$ de extracto se trató con $\mathrm{HCl}$ concentrado y se observó la formación de precipitado de color amarillo (18).

2. Flavonoides. Una pequeña cantidad de cada extracto se disolvió en $\mathrm{H}_{2} \mathrm{SO}_{4}$ y se observó coloración amarilla para flavonoides, naranja para flavonas y rojo-azuloso para chalconas (18).

3. Alcaloides. Prueba de Wagner: una fracción del extracto se trató con reactivo de ensayo de Wagner $[1,27 \mathrm{~g}$ de yodo y $2 \mathrm{~g}$ de yoduro de potasio en $100 \mathrm{ml}$ de agua] y se observó la formación de color rojizo precipitado marrón (18).

4. Saponinas. Una pequeña cantidad de cada extracto se agitó con agua y se observó la formación de espuma persistente por más de dos minutos (18). 
5. Triterpenos. Prueba de Liebermann-Burchard: se mezcló $1 \mathrm{~mL}$ de anhídrido acético y uno de cloroformo, se enfrió a $0^{\circ}$ y se le añadió gota a gota $\mathrm{H}_{2} \mathrm{SO}_{4}$. Se interpretó como positivo si ocurrió formación de colores azul, verde, rojo, anaranjado (18).

6. Fenoles. Prueba de cloruro férrico: Una fracción de cada extracto se trató con cloruro férrico al 5\% y se observó la formación del color azul o negro profundo (18).

Como controles se utilizaron aceites esenciales de eucalipto y tomillo.

\section{Separación y visualización de las fracciones por cromatografía en capa fina}

Para realizar este proceso se utilizó el extracto concentrado etanólico y se procedió a realizar una cromatografía para alcaloides, flavonoides, saponinas y triterpenos siguiendo el protocolo de Wagner (19). Los controles de las pruebas fueron adquiridos en el departamento de química de la Universidad $\mathrm{Na}$ cional y fueron específicos para cada cromatografía. Para Alcaloides se usó Efedrina, para flavonoides se utilizó Flavones (Flavonol), para saponinas se utilizó Saponinas purificadas y para triterpenos se utilizó Lupeol. La lectura de las cromatografías se realizó a una longitud de onda 365nm (luz U.V).

\section{Ensayo de la actividad antimicrobiana}

Para valorar la actividad antimicrobiana de los extractos etanólicos de Baubinia sp., Sambucus nigra,

Eichhornia crassipes y Taraxacum officinale se siguió el protocolo de la CLSI de susceptibilidad antimicrobiana por difusión en agar (20), por duplicado de cada parte de las plantas frente a ocho bacterias y una levadura. Se utilizaron cuatro concentraciones de cada extracto. Las cepas bacterianas ensayadas fueron: Streptococcus pneumoniae, Enterococcus faecium resistente a vancomicina, Klebsiella pneumoniae con presencia de KPC, Providencia rettgeri con presencia de ESBL, Pseudomonas aeruginosa ATCC 9027, Enterobacter cloacae, Escherichia coli, Staphylococcus aureus $\beta$-lisina ATCC 29213 y la cepa del hongo Candida albicans.

Como diluyente se utilizó Dimetil Sulfóxido (DMSO) para realizar la suspensión de los extractos y sus respectivas diluciones siguiendo el protocolo de Tello y colaboradores (17). La suspensión en DMSO se realizó debido a las características que posee de conservar principios activos en los medicamentos y facilitar su difusión en medios polares.

Se realizaron controles de crecimiento, esterilidad y de susceptibilidad antimicrobiana con antibióticos comerciales específicos para cada microorganismos ensayado.

\section{Resultados}

\section{Análisis Fitoquímico}

Tabla 1. Análisis fitoquímico obtenido de los extractos orgánicos.

\begin{tabular}{|l|c|c|c|c|c|c|c|c|c|c|c|c|}
$\begin{array}{c}\text { PLANTA / } \\
\text { ENSAYO }\end{array}$ & $\begin{array}{c}\text { Control } \\
\text { con aceites } \\
\text { escenciales }\end{array}$ & \multicolumn{4}{c|}{ Sambucus nigra } & \multicolumn{2}{c|}{$\begin{array}{c}\text { Eichhornia } \\
\text { crassipes }\end{array}$} & \multicolumn{2}{c|}{ Bauhinia sp } & \multicolumn{2}{c}{$\begin{array}{c}\text { Taraxacum } \\
\text { officinale }\end{array}$} \\
\hline & Control & Hojas & Tallos & Flores & Zumo & Hojas & Tallos & Hojas & Tallos & Hojas & Tallos \\
\hline Quinonas & +++ & ++ & ++ & +++ & - & - & + & - & + & +++ & +++ \\
\hline Flavonoides & +++ & +++ & +++ & +++ & +++ & - & + & - & +++ & ++ & + \\
\hline Alcaloides & +++ & +++ & - & +++ & ++ & - & - & - & ++ & - & - \\
\hline Saponinas & +++ & ++ & - & + & + & - & + & - & ++ & - & + \\
\hline Trierpenos & +++ & +++ & - & +++ & - & +++ & +++ & +++ & +++ & +++ & ++ \\
\hline Fenoles & +++ & - & - & - & +++ & - & - & - & - & - & - \\
\hline
\end{tabular}

+ Poca cantidad, ++ Moderada Cantidad, +++ Abundante cantidad, - No se evidencia reacción 
La Tabla 1 muestra los resultados obtenidos del análisis fitoquímico. Las hojas y flores de Sambucus nigra y los tallos de Bauhinia sp., tenían todos los grupos de complejos buscados a excepción de fenoles, mientras que por el contrario el único extracto de las plantas que tenía fenoles fue el zumo de Sambucus nigra. Los extractos que menos compuestos presentaron fueron los de las hojas de Eichhornia crassipes y Bauhinia sp., los cuales solo presentaron triterpenos. Según los resultados obtenidos mediante el análisis fitoquímico, se evidenció que los componentes más frecuentes en las plantas en estudio son los triterpenos, con presencia en ocho de las diez partes de las plantas analizadas; seguidos de los flavonoides, con el mismo número de test positivos, pero en menor cantidad.
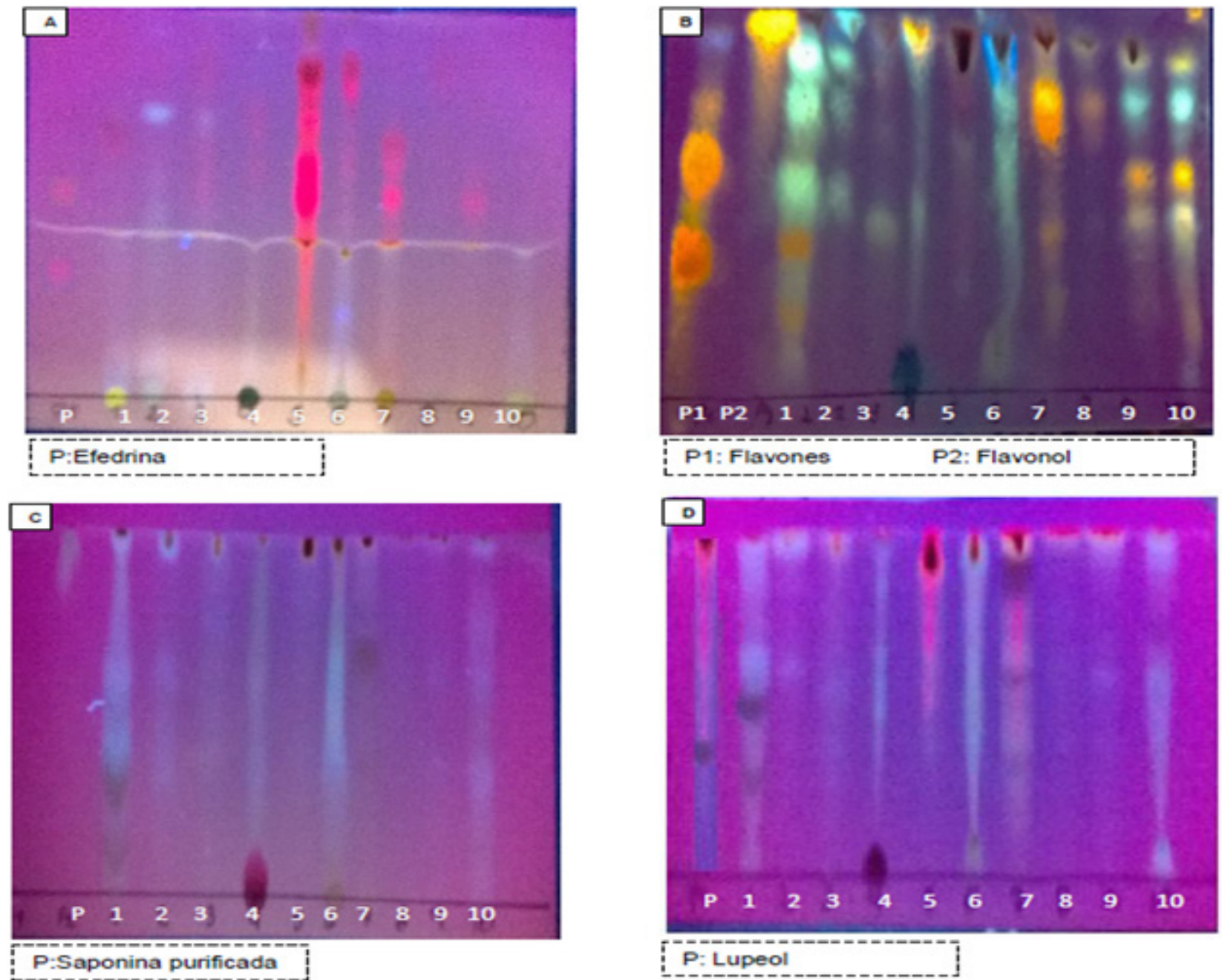

Figura 1. Cromatografías reveladas leídas en luz U.V. (365 nm) A. Alcaloides. B. Flavonoides. C. Saponinas. D. Triterpenos. P: Control 1: Sambucus nigra hojas, 2: Sambucus nigra tallos, 3: Sambucus nigra flores, 4: Sambucus nigra zumo, 5: Eichhornia crassipes hojas, 6: Eichhornia crassipes tallos, 7: Bauhinia sp. tallos, 8: Bauhinia sp. hojas, 9: Taraxacum officinale hojas,

10: Taraxacum officinale tallos. 
El análisis por cromatografía de capa fina para alcaloides, Figura 1A, evidenció que los tallos de T. officinale (posición 10) fueron los únicos que no tenían ningún tipo de compuesto perteneciente al grupo de los alcaloides. Para flavonoides (Figura 1B), la única muestra en donde no se relevaron flavonoides fue la correspondiente a las hojas de E. crassipes (posición 5). En el análisis de saponinas (figura 1C), las hojas de E. crassipes (posición 5) y hojas de Bauhinia sp. (Posición 8), fueron las únicas muestras en las que no se identificaron compuestos del tipo saponinas. Para el análisis de triterpenos (figura 1D) la cromatografía evidenció que todas las muestras presentaban por lo menos un compuesto de este tipo.

\section{Ensayos de actividad antimicrobiana por difusión en agar}

Tabla 2. Lectura de halos de inhibición obtenidos como resultado del ensayo de actividad antimicrobiana por difusión en agar.

\begin{tabular}{|c|c|c|c|c|c|c|c|c|c|c|}
\hline \multirow{2}{*}{$\begin{array}{l}\text { PLANTA Y } \\
\text { PARTE }\end{array}$} & \multirow{2}{*}{$\begin{array}{l}\text { CONCENTRACIÓN } \\
\text { DE EXTRACTOS }\end{array}$} & \multicolumn{9}{|c|}{$\begin{array}{l}\text { MICROORGANISMOS UTILIZADOS EN LAS PRUEBAS DE ACTIVIDAD ANTIMICROBIANA } \\
\text { HALOS DE INHIBICIÓN }(\mathrm{mm})\end{array}$} \\
\hline & & 1 & 2 & 3 & 4 & 5 & $\overline{6}$ & 7 & $\overline{8}$ & 9 \\
\hline \multirow{4}{*}{$\begin{array}{l}\text { T. officinale } \\
\text { tallos }\end{array}$} & $1000 \mu \mathrm{g} / \mu \mathrm{l}$ & 11,5 & 22 & 11 & 12 & 12 & 10,5 & 11 & 13 & 17 \\
\hline & $500 \mu \mathrm{g} / \mu \mathrm{l}$ & 11 & 15 & 10 & 11 & 10,5 & 10,5 & 10 & 12 & 11,5 \\
\hline & $250 \mu \mathrm{g} / \mu \mathrm{l}$ & 10 & 6 & 9,5 & 9,5 & 9 & 7,5 & 8 & 10 & 9 \\
\hline & $125 \mu \mathrm{g} / \mu \mathrm{l}$ & 9 & 6 & 9 & 8,5 & 7 & 6 & 6 & 8,50 & 6 \\
\hline \multirow{4}{*}{$\begin{array}{l}\text { T. officinale } \\
\text { hojas }\end{array}$} & $1000 \mu \mathrm{g} / \mu \mathrm{l}$ & 14,5 & 10 & 8,5 & 10,5 & 10,5 & 8,5 & 8,5 & 8,5 & 25 \\
\hline & $500 \mu \mathrm{g} / \mu \mathrm{l}$ & 10 & 9 & 9 & 9,5 & 8,5 & 7,5 & 7,5 & 7 & 12 \\
\hline & $250 \mu \mathrm{g} / \mu \mathrm{l}$ & 8 & 8 & 6 & 10,5 & 6 & 6 & 6 & 6 & 10,5 \\
\hline & $125 \mu \mathrm{g} / \mu \mathrm{l}$ & 6 & 6 & 6 & 9 & 6 & 6 & 6 & 6 & 9 \\
\hline \multirow{4}{*}{$\begin{array}{l}\text { E. crassipes } \\
\text { tallos }\end{array}$} & $1000 \mu \mathrm{g} / \mu \mathrm{l}$ & 12 & 20,5 & 7,5 & 11,5 & 18 & 11,5 & 8 & 12 & 12 \\
\hline & $500 \mu \mathrm{g} / \mu \mathrm{l}$ & 12 & 15,5 & 8,5 & 8,5 & 10 & 10 & 8 & 10 & 10 \\
\hline & $250 \mu \mathrm{g} / \mu \mathrm{l}$ & 8 & 6 & 6 & 6 & 8 & 9 & 9 & 6 & 9 \\
\hline & $125 \mu \mathrm{g} / \mu \mathrm{l}$ & 6 & 6 & 6 & 6 & 6 & 8 & 6 & 6 & 6 \\
\hline \multirow{4}{*}{$\begin{array}{l}\text { E. crassipes } \\
\text { hojas }\end{array}$} & $1000 \mu \mathrm{g} / \mu \mathrm{l}$ & 14 & 17,5 & 10 & 15 & 11,5 & 10 & 10,5 & 12,5 & 16,5 \\
\hline & $500 \mu \mathrm{g} / \mu \mathrm{l}$ & 11,5 & 12 & 9 & 10 & 10 & 9,5 & 9,5 & 10,5 & 15,5 \\
\hline & $250 \mu \mathrm{g} / \mu \mathrm{l}$ & 11 & 12 & 7 & 9 & 9 & 9 & 8 & 8 & 15 \\
\hline & $125 \mu \mathrm{g} / \mu \mathrm{l}$ & 10 & 6 & 6 & 9 & 8 & 6 & 7 & 6 & 11 \\
\hline \multirow{4}{*}{$\begin{array}{l}\text { Bauhinia sp. } \\
\text { Tallos }\end{array}$} & $1000 \mu \mathrm{g} / \mu \mathrm{l}$ & 11 & 6 & 9 & 10,5 & 9 & 9,5 & 9,5 & 12,5 & 12,5 \\
\hline & $500 \mu \mathrm{g} / \mu \mathrm{l}$ & 11 & 6 & 8 & 9 & 8,5 & 8,5 & 8,5 & 11,5 & 10 \\
\hline & $250 \mu \mathrm{g} / \mu \mathrm{l}$ & 6 & 6 & 6 & 7 & 8 & 7,5 & 6 & 10 & 9 \\
\hline & $125 \mu \mathrm{g} / \mu \mathrm{l}$ & 6 & 6 & 6 & 7 & 8 & 7 & 6 & 9 & 8 \\
\hline \multirow{4}{*}{$\begin{array}{l}\text { Bauhinia sp. } \\
\text { hojas }\end{array}$} & $1000 \mu \mathrm{g} / \mu \mathrm{l}$ & 12 & 14 & 11,5 & 13,5 & 10 & 8 & 10 & 8 & 11,5 \\
\hline & $500 \mu \mathrm{g} / \mu \mathrm{l}$ & 10 & 10 & 10 & 9 & 9 & 6 & 8 & 6 & 10 \\
\hline & $250 \mu \mathrm{g} / \mu \mathrm{l}$ & 6 & 6 & 8 & 7,5 & 7,5 & 6 & 8,5 & 6 & 6 \\
\hline & $125 \mu \mathrm{g} / \mu \mathrm{l}$ & 6 & 6 & 6 & 6 & 7 & 6 & 6 & 6 & 6 \\
\hline
\end{tabular}




\begin{tabular}{|c|c|c|c|c|c|c|c|c|c|c|}
\hline \multirow{4}{*}{ S. nigra tallos } & $1000 \mu \mathrm{g} / \mu \mathrm{l}$ & 10,5 & 13,5 & 7,5 & 12 & 6 & 10 & 8 & 16 & 15,5 \\
\hline & $500 \mu \mathrm{g} / \mu \mathrm{l}$ & 10 & 10 & 6 & 10,5 & 6 & 7,5 & 7,5 & 14,5 & 9,5 \\
\hline & $250 \mu \mathrm{g} / \mu \mathrm{l}$ & 8 & 9 & 6 & 9 & 6 & 8 & 7 & 13 & 9 \\
\hline & $125 \mu \mathrm{g} / \mu \mathrm{l}$ & 6 & 6 & 6 & 8 & 6 & 6 & 6 & 12,5 & 8 \\
\hline \multirow{4}{*}{ S. nigra hojas } & $1000 \mu \mathrm{g} / \mu \mathrm{l}$ & 10 & 15 & 6 & 8 & 10 & 9 & 9 & 15 & 13,5 \\
\hline & $500 \mu \mathrm{g} / \mu \mathrm{l}$ & 8 & 6 & 6 & 7 & 8 & 7 & 8 & 13 & 12 \\
\hline & $250 \mu \mathrm{g} / \mu \mathrm{l}$ & 6 & 6 & 6 & 6 & 6 & 6 & 6 & 10 & 10 \\
\hline & $125 \mu \mathrm{g} / \mu \mathrm{l}$ & 6 & 6 & 6 & 6 & 6 & 6 & 6 & 9,5 & 8,5 \\
\hline \multirow{4}{*}{ S. nigra flores } & $1000 \mu \mathrm{g} / \mu \mathrm{l}$ & 13 & 20,5 & 9 & 8 & 12 & 8 & 9,5 & 20 & 10,5 \\
\hline & $500 \mu \mathrm{g} / \mu \mathrm{l}$ & 12 & 17 & 7 & 8 & 10 & 8 & 8 & 17 & 9 \\
\hline & $250 \mu \mathrm{g} / \mu \mathrm{l}$ & 9 & 11 & 7 & 8 & 10 & 7 & 8 & 15 & 6 \\
\hline & $125 \mu \mathrm{g} / \mu \mathrm{l}$ & 8 & 6 & 6 & 6 & 10 & 7 & 6 & 14 & 6 \\
\hline \multirow{4}{*}{$\begin{array}{l}\text { S. nigra } \\
\text { zumo }\end{array}$} & $1000 \mu \mathrm{g} / \mu \mathrm{l}$ & 11 & 6 & 8 & 8 & 9 & 6 & 6 & 6 & 25 \\
\hline & $500 \mu \mathrm{g} / \mu \mathrm{l}$ & 9 & 6 & 7 & 8 & 8 & 6 & 6 & 6 & 19 \\
\hline & $250 \mu \mathrm{g} / \mu \mathrm{l}$ & 8 & 6 & 6 & 7 & 8 & 6 & 6 & 6 & 14 \\
\hline & $125 \mu \mathrm{g} / \mu \mathrm{l}$ & 6 & 6 & 6 & 6 & 7 & 6 & 6 & 6 & 6 \\
\hline
\end{tabular}

1: Streptococcus pneumoniae. 2: Enterococcus faecium resistente a la vancomicina. 3: Klebsiella pneumoniae con KPC (productora de carbapenemasa). 4: Providencia rettgeri ESBL (Betalactamasas de espectro extendido). 5: Pseudomonas aeruginosa. 6: Enterobacter cloacae 7: Escherichia coli. 8: Staphylococcus aureus. 9: Candida albicans. Medición dada en milímetros $(\mathrm{mm})$.

En los ensayos de susceptibilidad antimicrobiana por difusión en agar los resultados para S. pneumoniae se obtuvieron con el extracto de hojas de T. officinale a una concentración de $1000 \mu \mathrm{g} / \mu \mathrm{l}$ con un halo de inhibición de $14.5 \mathrm{~mm}$. El mejor extracto frente $E$. faecium resistente a vancomicina fueron los tallos de T. officinale a una concentración de $1000 \mu \mathrm{g} / \mu \mathrm{l}$ con un halo de inhibición de $22 \mathrm{~mm}$ de diámetro. Para $K$. pneumoniae con $K P C$ se observó que el extracto hojas Baubinia sp. fue el que presentó mayor efectividad en la concentración de $1000 \mu \mathrm{g} / \mu \mathrm{l}$ con una inhibición de $11.5 \mathrm{~mm}$. En el caso de $P$. rettgeri con ESBLs el extracto de hojas de $E$. crassipes fue el más efectivo a una concentración de $1000 \mu \mathrm{g} / \mu \mathrm{l}$ con un diámetro de inhibición de $15 \mathrm{~mm}$. P. aeruginosa presentó un halo de inhibición de $18 \mathrm{~mm}$ a una concentración de $1000 \mu \mathrm{g} / \mu \mathrm{l}$ del extracto de tallos de E. crassipes. E. cloacae fue inhibida con $11.5 \mathrm{~mm}$ a $1000 \mu \mathrm{g} / \mu \mathrm{l}$ con el extracto de tallos de E. crassipes. El extracto de tallos de T. officinale inhibió a $E$. coli con un halo de $11 \mathrm{~mm}$ a una concentración de $1000 \mu \mathrm{g} / \mu \mathrm{l}$. Para $S$. aureus el extracto de lores $S$. nigra resultó ser el más efectivo a una concentración de $1000 \mu \mathrm{g} / \mu \mathrm{l}$ inhibiéndolo con $20 \mathrm{~mm}$ de diámetro y fue efectivo hasta una concentración de $125 \mu \mathrm{g} / \mu \mathrm{l}$ con $14 \mathrm{~mm}$ de inhibición. C. albicans fue inhibida con un halo de $25 \mathrm{~mm}$ a una concertación de $1000 \mu \mathrm{g} / \mu \mathrm{l}$ del extracto zumo $S$. nigra efectivo hasta una concentración de $250 \mu \mathrm{g} /$ $\mu \mathrm{l}$ con $14 \mathrm{~mm}$ de inhibición.

\section{Discusión}

La resistencia bacteriana demostrada por investigadores de todo el mundo es el motivo por el cual en forma permanente se estén investigando nuevos compuestos, particularmente y en consideración a las investigaciones genéticas que han demostrado genes de resistencia en microorganismos (19-21). En el siglo XXI hay un buen número de estudios en donde la búsqueda se centra 
en diferentes recursos naturales, principalmente en las plantas, debido a la riqueza en compuestos con diferentes actividades, entre ellas la capacidad de inhibir el crecimiento de microorganismos $(22,23)$.

Los diversos mecanismos de acción antibacteriana de las plantas y los posibles metabolitos implicados han sido estudiados mediante ensayos de susceptibilidad antimicrobiana y análisis fitoquímicos debido a los potenciales usos contra las enfermedades infecciosas $(24,25)$. El presente estudio reveló la presencia de metabolitos secundarios como terpenoides, compuestos fenólicos, flavonoides, saponinas y alcaloides en los extractos de Bauhinia sp., $S$. nigra, T. officinale y E. crassipes (Tabla 1 y Figura 1). Algunos de los extractos derivados de las plantas analizadas presentaron actividades antimicrobianas relevantes, Tabla 2 .

Los estudios realizados demuestran que los metabolitos, en su mayoría secundarios, tales como alcaloides, flavonoides, taninos, y otros compuestos de naturaleza fenólica son responsables de las actividades antimicrobianas en plantas superiores $(26,27)$.

Los flavonoides son hidroxilados de sustancias fenólicas que suelen ser sintetizados por las plantas en respuesta a las infecciones microbianas (28), la actividad antimicrobiana de los flavonoides puede deberse a su habilidad para formar interacciones proteicas con proteínas intracelulares y por la interacción para la formación de complejos con las paredes celulares bacterianas que involucra la lisis celular (29).

Los terpenoides también han sido informados como antimicrobianos debido a la capacidad que poseen de causar una desestabilización en la integridad y permeabilidad de la membrana, al interferir con la disipación de la fuerza de los protones (30). Por lo tanto, la presencia de terpenoides y compuestos fenólicos podrían explicar la actividad antimicrobiana observada en el presente estudio.
Se ha demostrado la presencia de flavonoides, alcaloides, saponinas y triterpenos con actividad antimicrobiana en las plantas Fabáceas como Bauhinia sp., la cual ha sido comprobada frente a $E$. coli, $S$. aureus resistente a la meticilina y $K$. pneumoniae (31). Estos resultados pueden comparase con los obtenidos en este estudio, en los cuales se encontró una alta concentración de triterpenos en hojas y tallos de Baubinia sp. Además, en tallos se hallaron flavonoides, alcaloides y saponinas, los cuales previamente han sido reportados con una buena actividad antimicrobiana (32).

En el presente estudio, el extracto de Bauhinia sp. demostró ser efectivo a una concentración de $1000 \mu \mathrm{g} / \mu \mathrm{l}$ frente a $S$. pneumoniae, E. faecium y $P$. rettgeri con ESBL, $S$. aureus y $C$. albicans. Adicionalmente, las hojas demostraron ser eficaces frente a $K$. pneumoniae con $K P C$ a una concentración de $1000 \mu \mathrm{g} / \mu \mathrm{l}$ con una inhibición de $11,5 \mathrm{~mm}$ de inhibición, el más amplio reportado en el presente estudio para este patógeno de elevada resistencia y responsable de fallas en el tratamiento antibiótico convencional, Tabla 2. En un estudio realizado por Dhale (33) se encontró que extractos a partir de solventes orgánicos de Bauhinia a concentraciones de $20 \mathrm{mg} / \mathrm{ml}$, lograron inhibir $P$. aeruginosa y $E$. coli reportando un halo de inhibición de $10 \mathrm{~mm}$. Estos datos evidencian que los resultados obtenidos dependen de los métodos de extracción y solventes Por los cuales presentan afinidad química debido al comportamiento polar o apolar del solvente Además, es necesario analizar los resultados con otros métodos de extracción y otros solventes como la hidrodestilación asistida por microondas (34), o la destilación con fluido supercrítico (35). Igualmente, es recomendable realizar estos ensayos con microdiluciones en tubo para observar si hay actividad con menores concentraciones o se mantienen los mismos resultados a los obtenidos en difusión por agar.

En el caso de $S$. nigra se demostró que tiene varios de los compuestos analizados en cada una de las partes de la planta. En las hojas se observó un alto 
contenido de quinonas, flavonoides, saponinas y triterpenos, mientras que los tallos demostraron la presencia de quinonas y triterpenos; las flores presentaron quinonas, flavonoides, alcaloides y triterpenos y el zumo de bayas presentó flavonoides y fenoles, este último fue el más abundante en esta parte de la planta.

Dichos compuestos han sido descritos como los más probables agentes antimicrobianos en la familia Adoxaceae, de la cual hace parte S. nigra (36). Dos estudios han demostrado efectividad de esta planta como antiviral $(37,38)$; también ha sido ensayado como antibacteriano siendo muy eficaz frente a $S$. aureus resistente a la meticilina (39).

Los resultados obtenidos en esta investigación referente a $S$. nigra son similares a los hallazgos de Hearst y colaboradores (40), quienes demostraron la actividad antimicrobiana de las flores de $S$. nigra frente a $S$. aureus resistente a la meticilina. Según estos autores, la actividad de $S$. nigra podría ocurrir por compuestos fenólicos. En el presente estudio, se encontró que dependiendo de la parte de la planta analizada, esta presentaba una inhibición diferente frente a cada patógeno.

Los microorganismos que presentaron un mayor halo de inhibición con los extractos de $S$. nigra fueron $S$. aureus, E. faecium resistente a la vancomicina, $C$. albicans y $P$. rettgeri con presencia de ESBL a una concentración de $1000 \mu \mathrm{g} / \mu \mathrm{l}$. En la búsqueda realizada, no se encontraron reportes de actividad microbiana del zumo de las bayas de $S$. nigra, por lo tanto, el presente estudio sería uno de los primeros reportes en los que se encontró que las bayas tienen actividad antimicrobiana.

En un estudio reciente se demostró la capacidad de $S$. nigra frente a $S$. aureus ATCC 6538 a una concentración de $15 \mathrm{mg}$ con la técnica de microdilución (41). Este resultado lleva a que se proyecte a futuro un estudio utilizando microdilución en tubos para verificar la actividad con concentraciones bajas.
Con relación a la actividad antimicrobiana de $E$. crassipes, el trabajo realizado por Jayanthy y colaboradores (42) no encontró presencia de triterpenos, flavonoides, ni alcaloides, contrario a lo obtenido en este estudio, donde se demostró alta presencia de triterpenos y moderada de flavonoides y alcaloides. Al respecto, la investigación realizada por Kumar y colaboradores (43) demostró actividad antimicrobiana similar a la obtenida en este trabajo, pero con la diferencia de que los compuestos hallados en la planta fueron evaluados por dos técnicas diferentes (44), análisis fitoquímicos y cromatografía en capa fina y en ambas se encuentra la presencia de estos compuestos.

La actividad antimicrobiana hallada en esta planta demostró ser efectiva frente a $S$. pneumoniae, $E$. faecium resistente a la vancomicina, $P$. aeruginosa, S. aureus y C. Albicans. Este dato es similar al hallado por Kumar y colaboradores (42), quienes demostraron el efecto antimicrobiano de esta planta frente a Staphylococcus sp. Es importante el resultado obtenido del extracto de $E$. crassipes frente a $E$. cloacae porque se obtuvo un halo de inhibición de $10 \mathrm{~mm}$ de diámetro.

Los resultados arrojados por T. officinale demostraron que posee terpenos y quinonas; además, en las hojas de esta planta se evidenciaron flavonoides, dato interesante si se compara con lo hallado por Lateer y colaboradores (45), quienes encontraron terpenos y quinonas, pero no flavonoides. Los extractos de esta planta demostraron con la mayoría de los microorganismos halos de inhibición a una concentración de $1000 \mu \mathrm{g} / \mu \mathrm{l}$.

Con este resultado se puede afirmar que el extracto de tallos de T. officinale a una concentración de $1000 \mu \mathrm{g} / \mu \mathrm{l}$ se consideró como el más efectivo porque presentó las mayores inhibiciones de todo el estudio.

En los estudios realizados por Lateef y colaboradores (45-47), lograron inhibir el crecimiento de $E$. coli y $S$. aureus con una concentración de 
$200 \mathrm{mg} / \mathrm{ml}$, concentración muy alta en comparación con los resultados en este estudio.

Se encontró actividad de los extractos orgánicos de diversas partes de las plantas Baubinia sp., $S$. nigra, E. crassipes y T. officinale como agentes antibacterianos frente a patógenos de importancia clínica. A partir de estos resultados, se podría inferir que en estas plantas existen compuestos como terpenos, alcaloides, flavonoides, quinonas, saponinas y fenoles que podrían estar involucrados en la actividad antimicrobiana de estas plantas.

Los resultados obtenidos en el presente estudios corresponden a datos preliminares los cuales deben ser confirmados por técnicas más sensibles y evaluar la Concentración Mínima Inhibitoria de cada extracto. Además es necesario establecer los posibles metabolitos de cada planta que estaría ejerciendo la actividad antimicrobiana y comprobar las posibles acciones que estarían ejerciendo sobre los microorganismos. En conclusión, los extractos orgánicos de las plantas que se utilizaron en este estudio poseen propiedades antimicrobianas, las cuales podrían ser útiles a futuro en la búsqueda de nuevos agentes terapéuticos.

\section{Agradecimientos}

Agradecemos su colaboración en la realización del presente proyecto a la Universidad Colegio Mayor de Cundinamarca, en especial al Laboratorio Central y al Departamento de Química de la Universidad Nacional de Colombia.

\section{Referencias}

1. Köser C, Ellington M, Cartwright E, Gillespie S, Brown N, Farrington M, et al. Routine Use of Microbial Whole Genome Sequencing in Diagnostic and Public Health Microbiology. PLoS Pathogens. 2012; (8): 1-9

2. Ramírez L, Castillo A, Vargas A. Evaluación del potencial antibacterial in vitro de Croton lechleri frente a aislamientos bacterianos de pacientes con ulceras cutáneas. Nova. 2013; (11) 19: 51-63.
3. Roca W. Tendencias en el desarrollo de capacidades biotecnológicas e institucionales para el aprovechamiento de la biodiversidad en los países de la Comunidad Andina. Informe preparado para la Comisión Económica para América Latina y el Caribe (CEPAL) y la Corporación Andina de Fomento (CAF). 2004: 66-98.

4. Organización Mundial de la Salud. Estrategia mundial de la OMS para contener la resistencia a los antimicrobianos. 2001: 1-104.

5. Giraldo S, Bernal M, Robayo A, Pardo A, Molano L. Descripción del uso tradicional de plantas medicinales en mercados populares de Bogotá D.C. Nova. 2015. (13) 23. 75-82.

6. Duarte O, Velho L. La bioprospección como mecanismo de cooperación para la construcción de capacidades endógenas en ciencia y tecnología y análisis de las capacidades de Colombia para adelantar procesos de Bioprospección. VI Jornadas Latinoamericanas de Estudios Sociales de la Ciencia y la Tecnología. ESOCITE. 2006: 1-26.

7. Valdir C. Chemical Composition and Biological Potential of plants from the genus Bauhinia. Phytother Res. 2009; (23): $1347-54$.

8. The Wealth of India. A Dictionary of Indian Raw Materials and Industrial products. CSIR New Delhi. 1959; (2): 56-57.

9. Lorenzi H, Matos F. Plantas medicinais no Brasil nativas e exóticas. São Paulo. Instituto Plantarum. 2008; (2).

10. Guarrera P. Traditional antihelmintic, antiparasitic and repelenteuses of plants in central Italy. J Ethnopharmacol.1999; (68): 183-192.

11. Mane C, Arjun B, Pandurang A. Biosorption And Biochemical Study On Water Hyacinth (Eichhornia Crassipes) With Reference To Selenium. Archieves of Applied Science Research. 2011; 3(1): 222-229.

12. Jayanthi P, Lalitha P. Determination of the in vitro reducing power of the aqueous extract of Eichhornia crassipes (Mart.) Solms. J Pharm Res. 2011; (4): 4003-4005.

13. Aboul-Enein A, Al-Abd A, Shalaby E, Abul-Ela F, Nasr-Allah A, Mahmoud A Et al. Eichhornia crassipes (Mart) solms: From water parasite to potential medicinal remedy. Plant Signaling \& Behavior. 2011; 6(6): 834-836.

14. Dearing M, Mangione A, Karasov W. Plant secondary compounds as diuretics: An overlooked consequence. Am Zool. 2001; (41): 890-901

15. Tettey C, Ocloo A, Nagajyothi P, Lee K. An in vitro analysis of antiproliferative and antimicrobial activities of solvent fractions of Taraxacum officinale (Dandelion) leaf. Journal of Applied Pharmaceutical Science. 2014; 4 (03): 041-045.

16. Brango J. Búsqueda de compuestos Inhibidores de Quorum Sensing (IQS) a partir de extractos de origen natural. Primera Fase. Trabajo de grado presentado como requisito para optar por el título de Magister en Ciencias-Química. 2011. Universidad Nacional de Colombia. Facultad de Ciencias, Departamento de Química: 173. 
17. Tello E., Castellanos L., Arévalo C., Duque C. New cembranoid diterpenes from the caribbean sea whip Eunicea knighti. J. Nat. Prod. 2009; (72): 1595-1602.

18. Harborne J. B. Phytochemical Methods: A guide to modern techniques of plant analysis. Chapman and Hall. New York. 1973: 279

19. Wagner H, Bladt. S. Plant Drug Analysis. A Thin Layer Chromatography Atlas. 2a edition. Springer.2001.

20. CLSI. Clinical and Laboratory Standars Institute. Performance Standars for Antimicrobial Susceptibility Testing; Twenty-Fourth. Information Supplement. Enero de 2014: 230.

21. Bolivar Z, Poutou R, Carrascal A. Resistencia Antimicrobiana y a desinfectantes de Listeria spp. Nova. 2008; (6) 10. 201$218-$

22. Organización Mundial de la Salud. OMS. Estrategia de la OMS para la medicina tradicional 2012-2023. 2013: 75.

23. Rodríguez O, Andrade W, Díaz F, Moncada B. Actividad antimicrobiana de líquenes de la cuenca alta del rio Bogotá. Nova. 2015. (13) 23. 67-74.

24. Pesewu G, Cutler R, Humber D. Antibacterial activity of plants used in traditional medicines of Ghana with particular reference to MRSA. J Ethnopharmacol. 2008; 116(1): 102111.

25. Moreno Z, Martínez P, Figueroa J. Efecto antimicrobiano In vitro de propóleos argentinos, colombianos y cubano sobre Streptococcus mutans ATCC 25175. 2007. Nova; (5) 7: 70-75.

26. Pandey A. Anti-staphylococcal activity of a pan-tropical aggressive and obnoxious weed Parihenium histerophorus: an in vitro study. National Academy Science Letters. 2007; 30(11-12): 383-386.

27. Mahomoodally M, Gurib-Fakim A, Subratty A. Antimicrobial activities and phytochemical profiles of endemic medicinal plants of Mauritius. Pharmaceutical Biology. 2005; 43(3): 237-242.

28. Dixon RA, Dey P, Lamb C. Phytoalexins: enzymology and molecular biology. Adv Enzymol Relat Areas Mol Biol. 1983; (55):1-136.

29. Tsuchiya H, Sato M, Miyazaki T, Fujiwara S, Tanigaki S, Ohyama M, Et al. Comparative study on the antibacterial activity of phytochemical flavanones against methicillin-resistant Staphylococcus aureus. J Ethnopharmacol. 1996; 50(1): 27-34.

30. Sikkema J, de Bont J, Poolman B. Interactions of cyclic hydrocarbons with biological membranes. J Biol Chem. 1994; 269(11): 8022-8028.

31. Lopez J, Olmedo D, Prashad M. Cribado de la actividad antimicrobiana de plantas panameñas de la familia Fabaceae.Revista Médica de la Universidad de Costa Rica. 2015; 8 (2): 11-22.

32. Hamburger M, Cordell G, Tantivatana P, Ruangrungs I. Traditional medicinal plants of Thailand, VIII. Isoflavonoids of Dalbergia candenatensis. J nat prod. 1987; 50(4): 696-699.
33. Dhale, D.A. Phytochemical screening and antimicrobial activity of Bauhinia variegata Linn. J. ecobiotechnology. 2011; 3(9): 04-07.

34. Stashenko E, Jaramillo B, Martinez J. Comparision of different extraction methods for the analysis of volatile secondary metabolities of Lippia alba (Mill.) N. E. Brown. Grown in Colombia and evaluations of its in vitro antioxidant activity. J. Chromatogr. A. 2004; (1025): 93-103.

35. Gomez S, Mata N, Rodriguez A. Production of rose geranium oil using supercritical fluid extraction. J. Supercritical Fluids. 2007; (41): 50-60.

36. Borkataky M, Bhusan B, Saikia L. Antimicrobial Activity and Phytochemical Screening of Some Common Weeds of Asteraceae Family. Int. J Pharm Sci Rev Res. 2013; 23 (1): 116-120.

37. Matte AK, A.K. A.R, Mata P.T.G.Phytochemical screening and evaluation of the antibacterial activity of Sambucus nigra L. flower extracts (Caprifoliaceae). Rev. bras. plantas med. 2015; 4 (17): 1049-1054.

38. Mohammadsadeghi S, Malekpour A, Zahedi S, Eskandari F. The Antimicrobial Activity of Elderberry (Sambucus nigra L.) Extract Against Gram Positive Bacteria, Gram Negative Bacteria and Yeast. Research Journal of Applied Sciences. 2013; (8): 240-243.

39. Salehzadeh, A, Asadpour L, Naeemi A, Houshmand E. Antimicrobial Activity of Methanolic Extracts of Sambucus Ebulus and Urtica Dioica Against Clinical Isolates of Methicillin Resistant Staphylococcus Aureus. Afr J Tradit Complement Altern Med. 2014; 11 (5): 38-40.

40. Hearst C, McCollum G, Nelson D, Ballard L, Millar C, Goldsmith C, et al. Antibacterial activity of elder (Sambucus nigra L.) flower or berry against hospital pathogens. J Med Plant. 2010; 4(17): 1805-1809.

41. Salehzadeh A, Asadpour L, Sadat A, Houshmand E. Antimicrobial Activity of Methanolic Extracts of Sambucus Ebulus and Urtica Dioica Against Clinical Isolates of Methicillin Resistant Staphylococcus Aureus. Afr J Tradit Complement Altern Med. 2014; 11(5): 38-40.

42. Jayanthi P, Lalitha P. Antimicrobial activity of solvent extracts of Eichhornia crassipes (Mart.) Solms. Der Pharma Chemica, 2013; 5(3): 135-140.

43. Kumar S, Kumar R, Dwivedi A, Pandey AK. In vitro antioxidant, antibacterial, and cytotoxic activity and in vivo effect of Syngonium podophyllum and Eichhornia crassipes leaf extracts on isoniazid induced oxidative stress and hepatic markers. Biomed Res Int. 2014; (2014): 1-11.

44. Rodríguez O, Andrade W, Diáz F, Moncada B. Actividad antimicrobiana deliquenes de la cuenca alta del rio Bogotá. NOVA; 13(23): $65-72$.

45. Lateef O, Issah Y. Screening ethanolic and aqueous leaf extracts of Taraxacum officinale for in vitro bacteria growth inhibition. JPBMS. 2012; (20). 
46. Ramírez, L. C. C., et al. Solubilización de fosfatos: una función microbiana importante en el desarrollo vegetal. Nova. 2014; 12(21).

47. Rodríguez, O. E., Andrade, W. A., Díaz, F. E., \& Moncada, B. Actividad antimicrobiana de líquenes de la cuenca alta del rio Bogotá. 2015; Nova, 13(23). 